\title{
Spontaneous recovery of language in patients with aphasia between 4 and 34 weeks after stroke
}

\author{
WENDY LENDREM, NADINA B LINCOLN \\ From the Speech Therapy, Department, Nottingham Area Health Authority and Department of Health Care \\ of the Elderly, Nottingham, UK
}

SUMMARY The paper describes the spontaneous recovery of language abilities of 52 stroke patients who were aphasic for more than 4 weeks. These patients had been randomly allocated to receive no speech therapy and had been assessed at 6-weekly intervals after a stroke. There was improvement in language abilities over time. Age, sex and aphasia type were not related to the amount of improvement. An aphasic patient's level of language ability at 6 months could be predicted on the basis of the test score on the Porch Index of Communicative Ability at 4 weeks.

Detailed information on the spontaneous recovery of language following a stroke is of great importance to speech therapists. They need to be able to separate natural recovery from improvement due to intervention. Accurate prognosis for recovery with and without treatment is necessary in order to ensure the economic provision of therapy and to determine priorities in the selection of patients for treatment.

Previous studies of spontaneous language recovery following stroke have demonstrated that improvement occurs in at least the first 3 or 4 months. ${ }^{1-4}$ However, some authors present evidence that it continues for longer in at least some patients. $^{5-7}$ There is a need to establish the amount of recovery which occurs in individual patients and the length of time that it may be expected to continue.

Factors affecting the amount of recovery have also been investigated, though there are considerable discrepancies in the results obtained. Sex differences in the pattern of recovery were demonstrated by Basso et al, ${ }^{8}$ who found that females improve significantly more than males in oral expression though not in auditory comprehension. Others have found that sex has no influence on lan-

Address for reprint requests: Dr NB Lincoln, Stroke Research Unit, General Hospital, The Ropewalk, Nottingham NG1 6HA.

Received 24 July 1984 and in revised form 23 November 1984. Accepted 12 January 1985 guage recovery. ${ }^{39}$ There is reasonable consensus that age is of relatively minor importance ${ }^{31012}$ though a study by Sands $e a^{5}$ found greater improvement in younger patients. It has also been suggested that different types of aphasic patients show distinctive recovery patterns ${ }^{34}$ and, related to this, that patients with more severe aphasia make a worse recovery ${ }^{10-13}$ However, some of the differences suggested in recovery of different types of aphasics have not always been found. For example, Demeurisse et $a^{4}$ found no difference in recovery between Broca's and Wernicke's aphasics, though both improved more than global aphasics. Sarno and Levita ${ }^{6}$ found that in the first 6 months fluent aphasics improved more than non-fluent, who improved more than global aphasics, whereas in the second 6 months after stroke, fluent aphasics improved least and global aphasics the most.

Contradictory findings may be attributable, at least in part, to the study of small selected samples of patients, the inclusion of both treated and untreated patients and differences in the assessment methods. Information on the spontaneous recovery of language after stroke is scanty and inconsistent. Although the need for a large scale study of spontaneous language recovery has been recognised for some time ${ }^{35}$ it has not been forthcoming. We have collected information on spontaneous language recovery in a group of aphasic stroke patients as part of a randomised control trial of the effect of speech therapy.${ }^{14}$ In this study aphasic patients were randomly allocated to receive either twice weekly speech therapy for 24 weeks from 10 weeks after 
stroke or no speech therapy. No differences were found between the two groups of patients on measures of language ability. The extent of recovery and the factors affecting both the change in language abilities over time and the level of language achieved at 6 months have been investigated in a sub-group of the patients allocated to the no speech therapy group.

\section{Method}

\section{Patients}

164 aphasic stroke patients were allocated to the no treatment group of a study to evaluate the effectiveness of speech therapy. ${ }^{14}$ Of these 33 died, four moved too far away, 10 refused to attend appointments, six were too ill to attend and two were subsequently found to have tumours. In addition 16 patients were found to be unfit for full assessment at 4 weeks after stroke and an additional 23 patients were unfit at 10 weeks after stroke on the basis of a screening test. Results on five other patients had to be excluded, one because she was blind and therefore unable to complete the full language assessment, one because she was treated in error and three because they had missed the 4 week assessment through late identification. There were 13 patients who had recovered from their aphasia by 10 weeks after stroke and therefore were not included in the trial of speech therapy. Results on these patients are not included in the present study. They were aged 40 to 87 years (mean 67.31, SD 10.82 years) and there were six women and seven men. They had all reached the 85 th percentile on either the Verbal or the Overall Scale of the Porch Index of Communicative Ability (PICA). ${ }^{18}$

Of the 52 patients included in the present investigation, 32 were male and 20 female. They were aged 48 to 80 years (mean 67.0 years, SD 8.4 years). There were 10 Broca's, 13 Wernicke's, eight Conduction and 16 Anomic aphasics, as classified on the Boston Diagnostic Aphasia Examination (BDAE).$^{15}$ Of the five patients who could not be classified on this test, two were considered to be global aphasics and three were impossible to categorise.

\section{Procedure}

Each patient was screened for inclusion prior to 4 weeks after stroke. The Whurr Aphasia Screening Test ${ }^{16}$ was administered and those with very severe or minimal aphasia were excluded. The criteria for exclusion were a score of four or more on eight of the following sections: A10, 11, 12, 18, 19, 20, B11, 15, 26, 27 or if they failed more than one item on each or two items on any of the first four subtests A1 to A4. In addition patients scoring in categories " $c$ ", " $d$ " or " $e$ " of the following sections of the Frenchay Dysarthria Assessment: ${ }^{17}$ Reflex-swallow, Palate-in speech and Laryngeal-volume, pitch and prosody, were excluded.

Those patients who were included on the basis of the screening test were assessed at 4 weeks after stroke on the Porch Index of Communicative Ability (PICA) ${ }^{18}$ as a measure of language abilities. In addition, two measures of non-verbal ability were given, the Block Design subtest of the Wechsler Adult Intelligence Scale (WAIS) ${ }^{19}$ and copy- ing the Rey-Osterreith complex figure. ${ }^{20}$ These were included as possible prognostic indices of recovery. Relatives were asked to complete the Speech Questionnaire $(\mathrm{SQ})^{21}$ as a measure of functional speech.

Patients were reassessed at 6 weekly intervals. At 10, 22 and 34 weeks after stroke they were assessed on the PICA, Functional Communication Profile (FCP) ${ }^{22}$ and the SQ. At 16 and 28 weeks a shortened version of the PICA $^{23}$ was administered. At 16 weeks the $\mathrm{BDAE}^{15}$ profile of speech characteristics was also given in order to classify patients into categories of aphasia type.

The assessments were carried out by two speech therapists who did not know that the patients were not receiving speech therapy. All patients were told that their progress was being monitored and that they would be given treatment when they ceased to make satisfactory progress. Patients requiring treatment were offered speech therapy after the 34 week assessment. The inclusion of a no speech therapy group was considered ethically acceptable because there was considered to be reasonable doubt whether the speech therapy service available to these patients was effective.

\section{Results}

\section{Evaluation of change over time}

The pattern of spontaneous recovery between 4 and

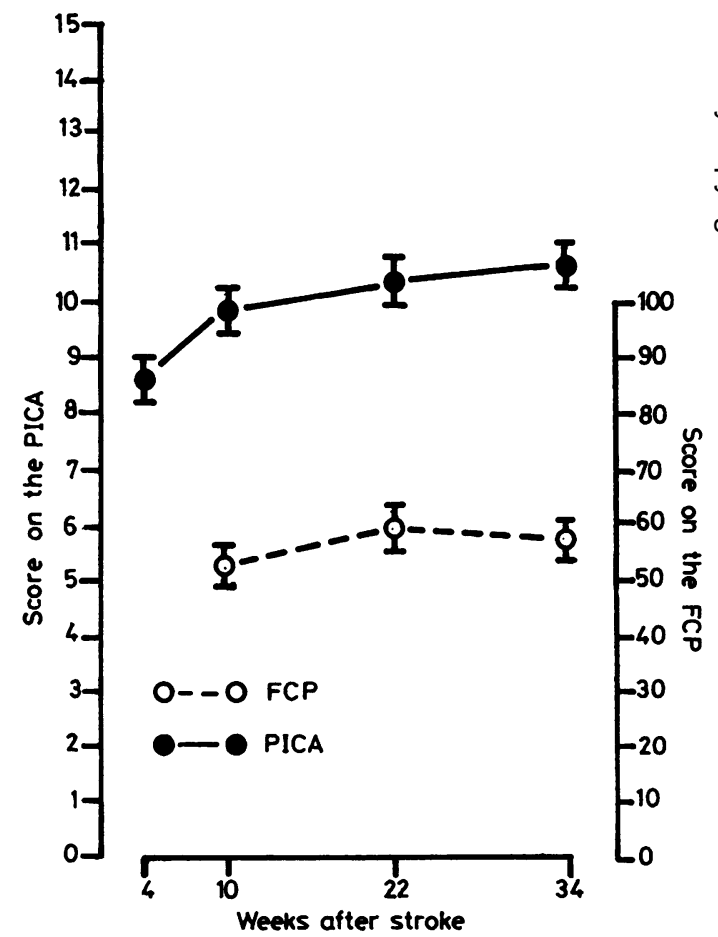

Fig 1 Mean and Standard Error of Mean on measures of language ability. 


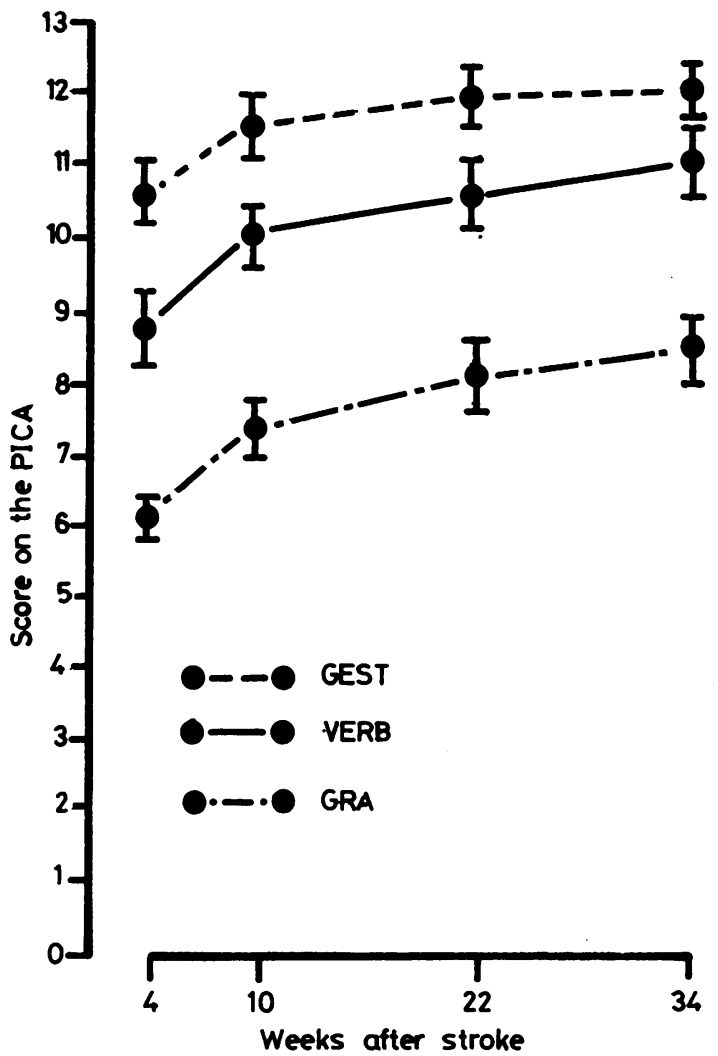

Fig 2 Mean and Standard Error of Mean on scales of the PICA.

34 weeks on the PICA is shown in fig 1. A one-way analysis of variance was carried out to determine whether there were differences between assessments. This indicated a significant difference between the four assessments in Overall PICA score $\left(F_{3,204}=7.62, p<0.001\right)$. A Scheffe procedure for grouping means showed that the 4 week assessment was significantly different from both the 22 and 34 week assessment but there were no significant differences between the 10 week and either the 22 or 34 week assessments.

Analyses of variance of the Verbal, Gestural and Graphics scales of the PICA also indicated significant differences between assessments for each of these scales (Verbal $F_{3,204}=4 \cdot 18, p<0.01$; Gestural $F_{3,204}=3.39$, $p<0.01$; Graphic $F_{3,204}=$ $10.85, p<0.001)$. The improvement on each of these scales is shown in fig 2 .

The recovery curves for individual patients in the main aphasia types are shown in figs 3 to 6 . It can be seen that although the mean PICA scores showed improvement from 4 to 34 weeks, some subjects deteriorated over this time especially between 22 and 34 week assessment. The percentage of subjects who improved and deteriorated is shown in the Table. This indicates that with time an increasing proportion of subjects deteriorated on their overall PICA scores.

The spontaneous recovery curve as measured with the FCP is also shown in fig 1 . There were no significant differences between 10,22 and 34 week FCP assessments $\left(F_{2,153}=1.46, p<0.25\right)$. The mean FCP scores showed that some recovery, although not statistically significant, occurred between 10 and 22 weeks, and there was a slight deterioration between 22 and 34 weeks. Analyses of variance for the dimensions of Movement, Speech, Understanding, Reading and Other (time, money, number) of the FCP also showed no significant differences between the 10, 22 and 34 week assessments (Movement $\mathrm{F}_{2,153}=0.89, \mathrm{p}>0.05$; Speech $F_{2,153}=0.89, p>0.05$; Understanding $F_{2,153}=$ $1.16, p>0.05$; Reading $F_{2,153}=2.38, p>0.05$; Other $F_{2,153}=2 \cdot 51, p>0 \cdot 05$ ).

There were 41 patients who had also completed a shortened version of the $\mathrm{PICA}^{23}$ at 16 and 28 weeks after stroke. The recovery curve of these 41 patients is shown in fig 7 .

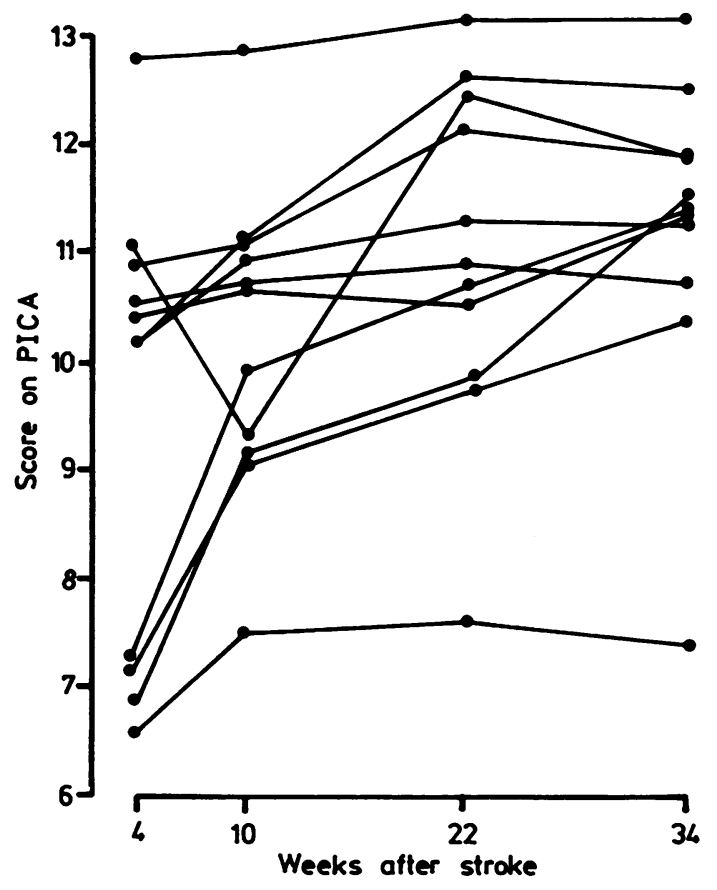

Fig 3 Spontaneous recovery of Broca's aphasics $(n=11)$. 


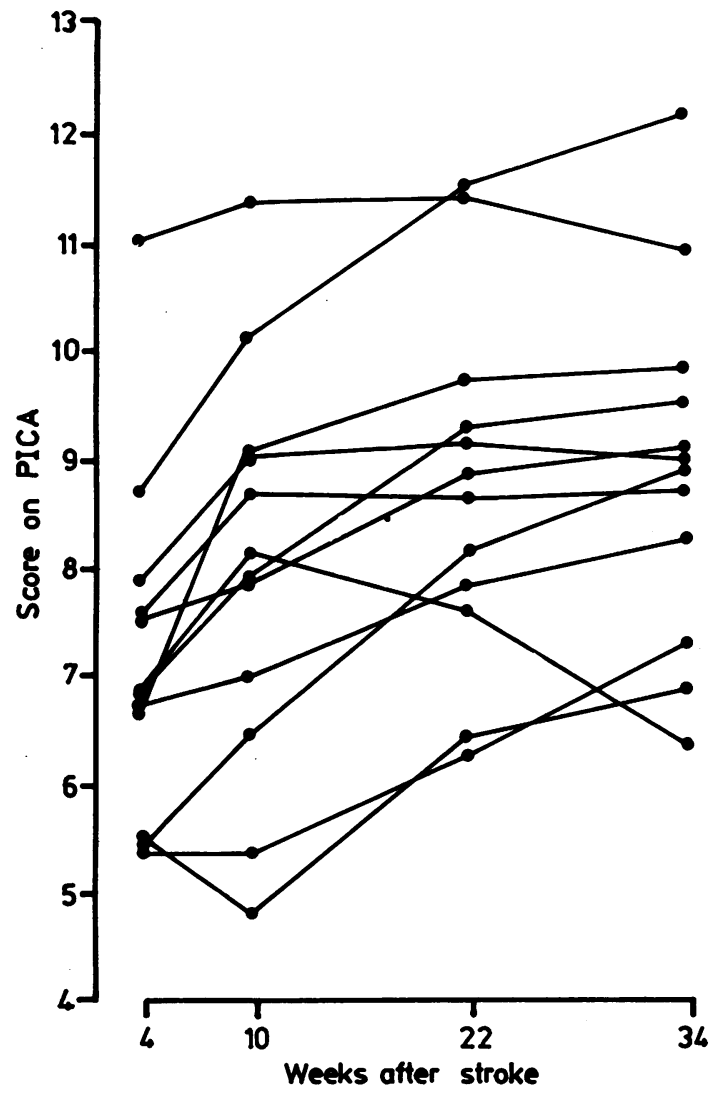

Fig 4 Spontaneous recovery of Wernicke's aphasics $(n=$ 12).

Factors affecting recovery

Analyses of variance (age decade $\times$ sex $\times$ type of aphasia) were carried out to determine the influence of these factors on both change in language abilities and the final level of language abilities reached at 34 weeks after stroke. None of the variables accounted for differences in the amount of change in language abilities between 4 and 34 weeks after stroke (Age $F_{4,40}=1 \cdot 83$, Sex $F_{1,40}=2 \cdot 36$, Type $F_{4,40}=9 \cdot 67$ ). However, there were significant differences between aphasia types and age decades in the PICA score reached at 34 weeks (Type $F_{4,40}=11 \cdot 13, p<0.001$; Age $\left.F_{4,40}=2.70, p<0.05\right)$. Broca's, Conduction and Anomic aphasics had significantly higher scores at 34 weeks than Wernicke's or global aphasics. The age group 60-69 years had significantly lower final level scores than other patients. Sex had no significant effect on final language level.

In order to determine possible predictors of spontaneous recovery from 4 weeks post onset, multiple
Table Proportion of subjects who improved or deteriorated in language abilities between assessments

\begin{tabular}{llll}
\hline & \multicolumn{3}{l}{ Weeks after stroke } \\
\cline { 2 - 4 } & 4 to 10 & 10 to 22 & 22 to 34 \\
\hline \% who improved & $90.4 \%$ & $78.9 \%$ & $63.5 \%$ \\
$\%$ who deteriorated & $7.7 \%$ & $21.2 \%$ & $36.5 \%$ \\
\hline
\end{tabular}

stepwise regression analyses were carried out for change in language abilities between 4 and 34 weeks and for the final language level at 34 weeks. The independent variables included in the analyses were age, scores on the 4 week PICA Verbal, Gestural and Graphic scales, and the measures of non-verbal ability: WAIS Block Design ${ }^{19}$ and Rey Figure Copying. ${ }^{20}$ These variables were all poor indicators of change in language ability between 4 and 34 weeks. All eight variables accounted for less than $7 \%$ of the variance. In contrast, the PICA Gestural, Graphic and Verbal scales at 4 weeks were good predictors of final language level reached and account for almost $69 \%$ of the variance. The prediction equation giving the closest fit to the data was:

Overall PICA score at 34 weeks $=3.86+(0.5 \times$ Gestural scale score $)+(0 \cdot 17 \times$ Graphics scale score $)+(1.25 \times$ Verbal scale score $)$
with the Gestural, Graphic and Verbal scales $\frac{\vec{\Phi}}{\omega} \vec{\omega}$ accounting for $65 \%, 4 \%$ and $3 \%$ of the variance in $\frac{}{\mathbb{\Phi}}$ 을 final scores respectively. Using the equation derived $\cong$ from these data, the 34 week post onset level of

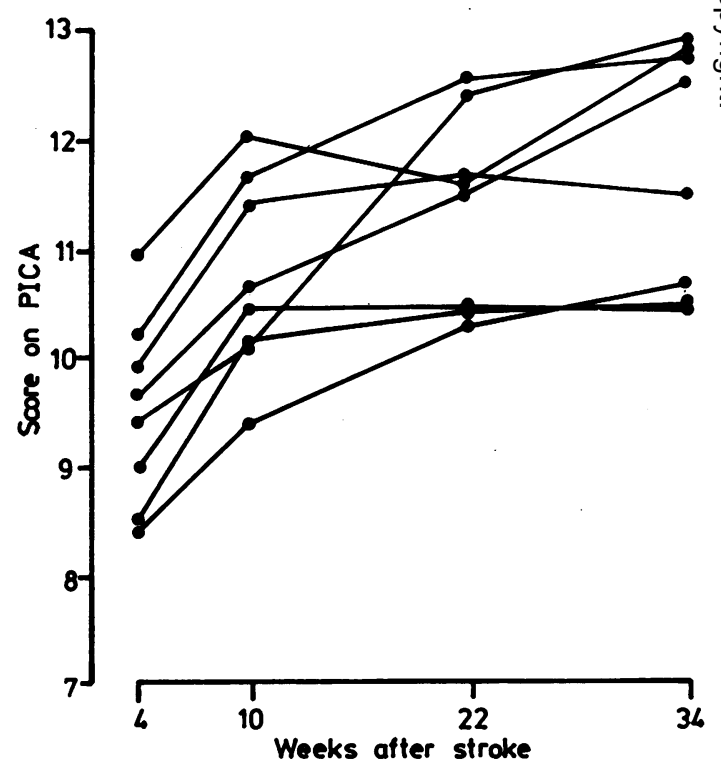

Fig 5 Spontaneous recovery of Conduction aphasics $(n=$ 8). 


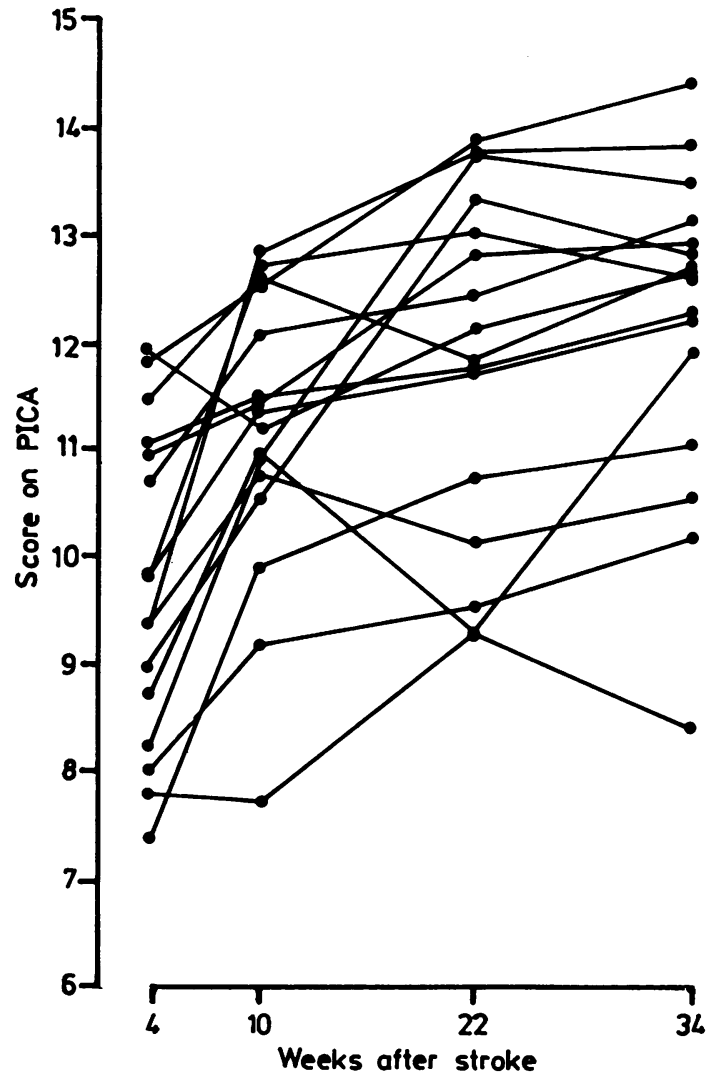

Fig 6 Spontaneous recovery of Anomic aphasics $(n=12)$.

language recovery can be predicted from the 4 week scale scores.

\section{Discussion}

The present results describe the spontaneous improvement of 52 aphasic patients. Although there were many more patients allocated to the no treatment group of the study, ${ }^{14}$ those patients included are likely to be representative of patients attending a speech therapy clinic. They were well enough and sufficiently concerned about their progress to attend out-patient appointments regularly for assessment. There were 13 patients who were recorded on admission as being aphasic yet by 4 weeks after stroke had recovered sufficiently to be unsuitable candidates for speech therapy. These 13 patients were not assessed later in the study but they had no characteristics which made them notably different from patients with aphasia persisting beyond 1 month after stroke.
The language abilities improved most markedly between 4 and 10 weeks after stroke and thereafter little change occurred. This is consistent with previous reports that most change is within the first three months, with little change thereafter. Even though the largest amount of change occurred between 4 and 10 weeks these two assessments were not statistically significantly different from each other, though the 4 week assessment was significantly different from both the 22 and 34 week assessment. If the mean values for the group are considered then the improvement in terms of clinical significance is not great. However, the changes of some individuals during this period were quite marked and would be of practical significance. There were also nine patients who improved by as much as two PICA points between 10 and 34 weeks after stroke, even though the group mean shows no change. This lack of change between these two assessments could be due to the increasing proportion of patients who deteriorate. The patients who deteriorated only tended to get worse by a small amount and there was only one patient who deteriorated by as much as 2 PICA points, this being due to a probable further stroke. In addition, five patients showed deterioration between two assessments of one PICA point, in four of these it was associated with deterioration in their physical condition and in one it was unexplained.

The lack of difference in change scores between either sex, age decades or aphasia types suggests that the amount of improvement expected in any patient cannot readily be predicted. The $F$ values

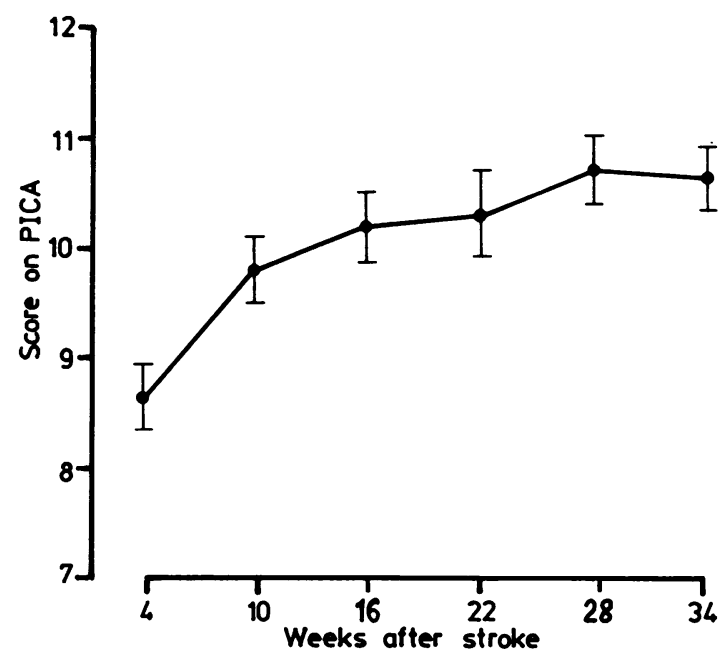

Fig 7 Mean and Standard Error of Mean on the Overall PICA for 41 patients with complete assessments. 
obtained were low indicating the differences did not even approach statistical significance. This is borne out by the regression analysis which showed that only a small proportion of the variance in change scores could be accounted for by the initial level of language abilities. In contrast, age, aphasia type and initial level of language abilities were associated with the level of language abilities at 34 weeks after stroke. This discrepancy in findings could result if everyone improves by a similar amount regardless of age, aphasia type or initial severity. However, there is considerable variation in change scores between 4 and 34 weeks (mean change $1.98, \mathrm{SD} 1 \cdot 31$, range -0.49 to $5 \cdot 18$ ) which does not support this explanation. Although subjective impressions are that younger patients improve more this was not supported by the present findings. It could be that grouping patients according to age decades masked any age effect. However, age decades were found to be different when the level of abilities reached was considered, which suggests that this is an unlikely explanation for the lack of agreement between our results and subjective impressions.

In practical terms, at 4 weeks post stroke a speech therapist should be able to predict a patient's level of functioning at 6 months simply on the basis of their test scores on the PICA. Although both aphasia type and severity were separately found to be associated with different levels of ability, this may simply reflect that certain aphasia types are associated with severe language impairment and others with mild. The two variables are confounded and it is not possible to separate them in the present study.

Sex differences were not observed in either the amount of change or the final level reached. This applied on PICA subtests as well as the summary scales, Verbal, Gestural and Graphic. This is inconsistent with the findings of Basso et al ${ }^{8}$ possibly because of differences in the method of selection of patients.

The present results support previous reports that most recovery occurs in the first three months after stroke, though there is some variation between patients. Nevertheless the amount of change seems to be relatively independent of the age or sex of the patient. The level of language abilities at six months post stroke seems to depend almost exclusively on the severity of the aphasia early after the stroke.

We thank the following for their assistance with this study: Dr GP Mulley, Prof JRA Mitchell, Miss M Edwards and Mrs E McGuirk for setting up the trial of speech therapy from which the data were obtained; Mrs E McGuirk for carrying out some of the language assessments; Mrs C Durow for clerical assistance; and The Research Committee of Trent RHA for financial support.

\section{References}

' Culton GL. Spontaneous recovery from aphasia. J Speech Hear Res 1969;12:825-32.

${ }^{2}$ Sarno MT, Levita E. Natural course of recovery in severe aphasia. Arch Phys Med Rehabil 1971;52:175-9.

${ }^{3}$ Kertesz A, McCabe P. Recovery patterns and prognosis in aphasia. Brain 1977; 100: 1-18.

${ }^{4}$ Demeurisse G, Demol O, Derouck M, de Beuckelaer R, Cockaerts MJ, Capon A. Quantitative study of the rate of recovery from aphasia due to stroke. Stroke 1980;11:455-8.

${ }^{5}$ Sands E, Sarno MT, Shankweiller D. Long term assessment of language function in aphasia due to stroke. Arch Phys Med Rehabil 1969; 50:202-6.

${ }^{6}$ Sarno MT, Levita E. Recovery in treated aphasia in the first year post-stroke. Stroke 1979;10:663-9.

${ }^{7}$ Hanson WR, Cicciarelli. The time, amount and pattern of improvement in adult aphasics. Br J Disord Commun 1978;13:59-63.

${ }^{8}$ Basso A, Capitani E, Moraschini S. Sex differences in recovery from aphasia. Cortex 1982;18:469-75.

" Sarno MT, Silverman M, Levita E. Psychosocial factors and recovery in geriatric patients with severe aphasia. J Am Geriatr Soc 1970;13:405-9.

${ }^{10}$ Porch BE, Collins M, Werz RT, Frieden TP. Statistical prediction of change in aphasia. $J$ Speech Hear Res 1980;23:312-21.

" Hartman J. Measurement of early spontaneous recovery from aphasia with stroke. Ann Neurol 1981;9:89-91.

12 Pickersgill MJ, Lincoln NB. Prognostic indicators and the pattern of recovery of communication in aphasic stroke patients. J Neurol Neurosurg Psychiatry 1983;46: 130-9.

${ }^{13}$ Keenan JS, Brassel EG. A study of factors related to prognosis for individual aphasic patients. $J$ Speech Hear Disord 1974;39:257-69.

${ }^{14}$ Lincoln NB, McGuirk E, Mulley GP, Lendrem W, Jones AC, Mitchell JRA. The effectiveness of speech therapy for aphasic stroke patients: a randomised control trial. Lancet 1984;1:1197-200.

is Goodglass H, Kaplan E. The Assessment of Aphasia and Related Disorders. Boston: Lea and Febiger, 1972.

${ }^{16}$ Whurr R. The Whurr Aphasia Screening Test. Available on request from $\mathbf{R}$ Whurr, 2 Alwyne Road, London N1 $2 \mathrm{HH}$, UK.

17 Enderby P. The Frenchay Dysarthria Assessment. Windsor: NFER-Nelson, 1984.

18 Porch BE. The Porch Index of Communicative Ability. Palo Alto: Consulting Psychologists Press, 1967.

19 Wechsler D. The Wechsler Adult Intelligence Scale. New York: Psychological Corporation, 1955.

20 Rey A. Le test de copie de figure complexe. Paris: Editions de Psychologie appliquée, 1959.

${ }^{21}$ Lincoln NB. The Speech Questionnaire: an assessment of functional language ability. Int Rehab Med 1982;4:114-7.

${ }^{22}$ Sarno MT. The Functional Communication Profile: manual of directions. New York: Rehabilitation Monograph 42, Institute of Rehabilitation Medicine, 1969.

${ }^{23}$ Linclon NB, Ells P. A shortened version of the PICA. Br $J$ Disord Commun 1980; 15:183-7. 